\title{
Preserving and reserving the best for last: longevity begets wisdom
}

\author{
David F. Albertini ${ }^{1}$
}

Published online: 14 October 2015

(C) Springer Science+Business Media New York 2015

The recent passing of Howard Jones closes a chapter in the history of reproductive medicine in the USA and abroad. He will always be remembered for the wisdom he imparted on our profession immediately and for sharing with so many the courage and determination that was required to bring ARTs into the mainstream of infertility treatment in the USA. Besides being a mentor to the present day leaders in our field, his longevity carved a legacy that touched the lives of so many over his years at Johns Hopkins and through his tenure at the Eastern Virginia Medical School. Among those touched most profoundly by Howard and Georgeanna, none compares with their influence on the hopes and aspirations of Elizabeth Comeau. It is well worth it for our readers to have a look at the touching eulogy she proffers to Dr. Howard in which she recounts his lifelong influence on the first baby born by IVF in the USA (www.bostonglobe.com/news/science/2015/08/01/ the-first-ivf-baby-remembers-howard-jones/ 9MkRw23tcevVkZWVuVAZqI/story.html).

It is a well-known fact to most of our readership that the roots of reproductive endocrinology and human embryology were melded through the unique union of Georgeanna and Howard Jones. What may be less apparent, but equally relevant to the subject of preserving their legacy, is a brief but

Capsule The passing of Howard Jones closes an era in reproductive medicine raising important issues regarding how human ARTs evolved to this point and what the founders of this branch of medicine would think about the emergence and implementation of high technology accoutrements.

David F. Albertini

DALBERTINI@kumc.edu

1 University of Kansas Medical Center, Kansas, KS, USA significant event that took place in their laboratories in the middle 1960s. Over a matter of a few months during the summer of 1965 in Baltimore, Bob Edwards conducted pioneering experiments on the in vitro maturation (IVM) of human oocytes using clinical samples the Joneses recognized and encouraged would be vital to expanding our basic knowledge of human reproduction. Oologists of years gone by, and even those who today search for a niche for IVM in clinical practice, remain in awe of the publications that resulted from this work (in Nature and Lancet) not only because the studies provided the first clear evidence that human oocytes and those of other mammals could undergo meiotic maturation ex vivo, but they also established the fundamental concept that intrafollicular factors inhibited the resumption of meiosis, a notion initially proposed by Gregory Pincus in the 1930s. That oocytes could recapitulate in vitro what was happening in response to the LH surge in vivo was indeed prescient as Edwards and the Joneses first came to appreciate the possibilities for IVF.

With the marriage of the clinician-scientists cemented in what would become a lasting collaboration and friendship, it was only a matter of time, a measure of good luck, and a quantum of determination before human ARTs as we know it today would change the practice of reproductive medicine. Preserving, and reserving, the dignity and integrity exhibited by our forebears does indeed maintain the mindfulness sought as longevity begets wisdom. But how true to this legacy does human ARTs hold today?

Are we on a course where the best efforts aimed at treating infertility today will derive from an accumulation of knowledge, rendered as objective as possible, or from technologies bearing more than an altruistic (sic commercial) sense of purpose? So it is that in this issue of JARG we continue to see that the latest developments on the front of cryopreservation may not in fact meet the needs of patients who by virtue of 
age or other clinical parameters are best served by the status quo of everyday practice.

With respect to the growing diversity in ages of patients seeking treatment, two papers this month take on the matter of global perspectives in attempting to reveal patterns of care and outcomes with respect to ovarian reserve evaluation. Both antral follicle counts and AMH levels have been pronounced as biomarkers for some time now, and their reliability will continue to dominate discussions regarding variance within populations as a matter of chronological age (see Christianson and colleagues, Measurement of antral follicle counts in patients undergoing in vitro fertilization treatment: results of a worldwide web-based survey, DOI 10.1007/s10815-015-0555-6; Tobler et al., Use of anti-mullerian hormone for testing ovarian reserve: a survey of 796 infertility clinics worldwide, DOI 10-1007/s10815-015-0562-7) or conditions predisposing individuals as outliers due to iatrogenic (see Chan and colleagues, Outcomes of ovarian stimulation after treatment with chemotherapy, DOI 10.1007/s10815-015-0575-2) or what we have come to understand may be complex genetic factors confounded by lifestyle patterns.

As alluded to above, and as illustrated in our recent issue devoted to fertility preservation, the topic of cryopreservation continues to raise the level of discourse. Remaining at the heart of matters chilling, is the freeze-all promotion relative to the fresh transfer options subserving the mainstay of practices, until somewhat recently. Once again, what is the best strategy to counsel patients will depend on age, among other factors (Nicopolous et al., Treatment and preservation at the extremes of reproductive age: a case report outlining the ethical dilemmas, DOI 10.1007/s10815-015-0551-x).

We encourage our readership to contribute to the preservation of traditions and at the same time take pause when considering the latest advances in ART-related technology. Being vigilant and conservative in how costs are counterbalanced should not in the end compromise the quality of care if, as Howard Jones would have proclaimed, reserving judgment is a sign of wisdom enacted on behalf of the patient. 